\title{
Consumo de substancias durante el embarazo y dimensiones de personalidad
}

\section{Substance use during pregnancy and personality dimensions}

\author{
María Luisa Imaz*a , Ricard Navinés*a, Estel Gelabert**, Francina Fonseca***, \\ Alfonso Gutierrez-Zotes****, Roser Guillamat*****, Klaus Langohr******, \\ Francesca Canellas $* * * * * * *$, Inmaculada Gornemann*********, Javier Costas*********, \\ Fermín Mayoral $* * * * * * * *$, Vicens VAlles*****, Yolanda De Diego********, MiQuel Roca*******, \\ José Luis Iborra***********, Mónica Gratacos***********, Elisabeth Vilella****, \\ Lluïsa García-Esteve*, Julio Sanjuan**********, Marta Torrens***, Rocio Martin-Santos*.
}

* Servicio de Psiquiatría y Psicología, Unidad de Psiquiatría Perinatal, Hospital Clinic, Institut d’Investigació Biomèdica August Pi i Sunyer (IDIBAPS), Centro de Investigación en Red en Salud Mental (CIBERSAM), y Departmento de Medicina, Instituto de Neurociencias, Universidad de Barcelona (UB), Barcelona, Spain; ** Departmento de Psicología Clínica y de la Salud, Facultat de Psicología, Universitat Autònoma de Barcelona (UAB), Bellaterra (Barcelona), Spain; *** Grupo de Investigación en Adicciones, IMIM-Institut Hospital del Mar d'Investigacions Mèdiques, Parc de Salut-Mar, UAB, Red de Trastornos Adictivos (RediTA), Barcelona, Spain; **** Hospital Universitari Institut Pere Mata, IISPV, Universitat Rovira i Virgili. CIBERSAM. Institut Pere Mata, Reus, Spain; ***** Department of Mental Health, Consorci Sanitari de Terrassa, Terrasa (Barcelona), and Corporació Sanitaria de Parc Tauli, Sabadell (Barcelona), Spain; ****** Departament d'Estadística i Investigació Operativa, Universitat Politècnica de Catalunya, Barcelona, Spain; ******* Hospital Juan March, and Institut Universitari d'Investigació en Ciències de la Salut, Red de Asistencia Primaria (RediAPP, Palma de Mallorca, Islas Baleares, Spain; ******** Fundación de Investigación Biomédica de Málaga (IBIMA), and UGC Salud Mental. Hospital Regional Universitario de Málaga, Málaga, Spain; ********* Instituto de Investigación Sanitaria de Santiago de Compostela (IDIS) Servizo Galego de Saúde (SERGAS), Complexo Hospitalario Universitario de Santiago (CHUS). Santiago de Compostela, Spain; **********Hospital Clínico, University of Valencia, CIBERSAM, Valencia, Spain; *********** Centro de Regulación Genómica (CRG) y UPF, Barcelona, España, Centro de Investigación Biomédica en Red de Epidemiología y Salud Pública (CIBERESP), Barcelona, Spain.

a Ambos autores son primeros firmantes.

\section{Resumen}

Este estudio evalúa los patrones de consumo de substancias durante el embarazo y las dimensiones de personalidad asociadas, en una muestra multicéntrica de 1804 mujeres de población general. En el 2-3 día posparto, completaron una entrevista auto-administrada sobre el consumo de alcohol, tabaco, cafeína, cannabis, cocaína, opiáceos, drogas de diseño, además de variables socio-demográficas, obstétricas/reproductivas, historia psiquiátrica previa, apoyo social durante el embarazo y el cuestionario de personalidad de Eysenck (EPQ-RS). Se generaron modelos de regresión logística múltiple. La prevalencia del consumo fue del $50 \%(\mathrm{~N}=909)$ : $40 \%$ cafeína, $21 \%$ tabaco, 3,5\% alcohol, y 0,3 cannabis. Las puntuaciones $T$ medias $(D E)$ de personalidad fueron: extraversión 51,1 (9,6), psicoticismo $48(8,9)$ y neuroticismo $43,6(8,5)$. Las dimensiones de extraversión $(p=0,029)$ y psicoticismo $(p=0,009)$, fueron identificadas como factores de riesgo tras ajustar por edad, nivel educación, estatus laboral durante el embarazo, bajo apoyo social, e historia psiquiátrica previa. Para cada incremento de 10 unidades en sus

\begin{abstract}
Our aim was to assess personality traits associated with substance use during pregnancy in a population-based, multicentre study of 1804 pregnant women. On day 2-3 postpartum, participants completed a semi-structured interview, including self-reported drug use (alcohol, tobacco, caffeine, cannabis, cocaine, opioids) during pregnancy, and socio-demographic, reproductive and obstetric variables, personal and family psychiatric history, social support, and the Eysenck personality questionnaire, short version (EPQ-RS). Logistic regression models were conducted. Fifty per cent of women reported substance use during pregnancy: $40 \%$ caffeine, $21 \%$ tobacco, $3.5 \%$ alcohol, and 0.3 $\%$ cannabis. Mean $T$-scores $(S D)$ for personality dimensions were 51.1 (9.6) for extraversion, 48 (8.9) for psychoticism, and 43.6 (8.5) for neuroticism. Extroversion $(p=.029)$ and psychoticism $(p=.009)$ were identified as risk factors after adjustment by age, level of education, employment status during pregnancy, low social support, and previous psychiatric history. For each increment of 10 units in their scores,
\end{abstract}

Enviar correspondencia a: Rocio Martin-Santos. Servicio de Psiquiatría y Psicología, Unidad de Psiquiatría Perinatal, Hospital Clínic, Institut d'Investigació Biomèdica August Pi i Sunyer (IDIBAPS), Centro de Investigación en Red en Salud Mental (CIBERSAM), y Departamento de Medicine, Instituto de Neurociencias, Universidad de Barcelona, 08036-Barcelona, Spain. Phone: +34 932275400; Fax: +34 932275454. E-mail: rmsantos@clinic.cat. 
puntuaciones, el odds de consumo de substancias durante el embarazo se incrementó un $12 \%$ y un $16 \%$ respectivamente. Menor educación, estar de baja, y antecedentes psiquiátricos fueron también factores independientes $(p<0,05)$ asociados al consumo. Ser primípara fue factor protector $(p=0,001)$. El modelo final mostró un ajuste satisfactorio $(p=0,26)$. El cribaje de las mujeres con riesgo de consumo de substancias durante el embarazo debería incluir la personalidad además de variables psicosociales y antecedentes psiquiátricos. Identificar los factores de riesgo asociados es importante para prevenir y mejorar la salud materna y fetal/neonatal durante el embarazo y posparto.

Palabras clave: Alcohol; Cafeína; Cannabis; Tabaco; Consumo de substancias; Embarazo; Personalidad.

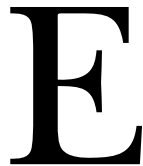

1 uso de substancias psicoactivas está ampliamente extendido entre la población general. A nivel mundial las substancias legales e ilegales más utilizadas son la cafeína, el tabaco, el alcohol y el cannabis (Kuczkowski, 2009; WHO, 2010). La prevalencia de consumo de substancias de uso recreacional entre los jóvenes, incluyendo las mujeres en edad de procrear (15-44 años), se ha incrementado de forma notable en las últimas dos décadas (Chen y Jacobson, 2012; EMCDD, 2016; SAMHSA, 2013). El consumo y sus efectos sobre el organismo son diferentes en las mujeres que los hombres (Ait-Daoud et al., 2019; Díaz-Mesa et al., 2016; Patró-Hernandez et al., 2019), siendo las mujeres más vulnerables al consumo de substancias durante la edad reproductiva (Louw, 2018).

El embarazo es un periodo singular en la mujer, en el que el uso de substancias puede afectar su salud así como interferir a diferentes niveles (teratogénico y epigenético) en el desarrollo del feto, dependiendo de la dosis, el tiempo y la substancia consumida (Álvarez-Segura et al., 2014; Cook et al., 2017; Volkow, Compton y Wago, 2017).

En las mujeres embarazadas, la prevalencia de consumo parece ser significativamente menor en comparación con las mujeres no embarazadas de igual edad (Lamy y Thibaut, 2010; Lange, Probst, Rehm y Popova, 2018; Popova, Lange, Probst y Rehm, 2017). En general, ésta se estima por extrapolación de estudios epidemiológicos en población general (Lange et al., 2018; Powers, McDermott, Loxton y Chojenta, 2013). Una revisión sistemática con meta-análisis (Lange et al., 2018) señala que a nivel global (131 países) el 53\% de la mujeres que fuman lo siguen haciendo durante el embarazo. Europa en concreto, muestra la mayor prevalencia de consumo de tabaco durante el embarazo, $8,1 \%$, pero la menor proporción de mujeres, $30 \%$, de consumidoras diarias. En España esta extrapolación apunta a una prevalencia de consumo de tabaco $\geq 20 \%$ durante el embarazo. El consumo de alcohol se sitúa en torno a un $10 \%$ variando según los países (Popova et al., 2017). En Europa se estima una prevalencia del $25 \%$ y en nuestro país entre un 10-15\% (Popova et al., 2017). Con respecto the odds of substance use increased by $12 \%$ and $16 \%$ respectively. Low education, being on leave during pregnancy, and previous psychiatric history were independent factors $(p<.05)$ associated with substance use during pregnancy. Primiparity was a protective factor $(p=.001)$. The final models showed a good fit $(p=.26)$. The screening of substance use during pregnancy should include personality dimensions apart from psychosocial variables and history of psychiatric disorders. It is important to identify the associated risk factors for substance use during pregnancy to prevent and improve foetal/neonatal and maternal health during perinatal period.

Keywords: Alcohol; Caffeine; Cannabis; Tobacco; Substance use; Pregnancy; Personality.

a la drogas ilegales, las dos substancias mas prevalentes durante el embarazo son el cannabis y la cocaína (Cook et al., 2017). Se calcula una prevalencia entre un 2\%-10\%, siendo mayor al inicio del embarazo y menor en el último trimestre (Alshaarawy y Anthony, 2019); y la de la cocaína en un 0,5-3\% (Bhuvaneswar, Chang, Epstein et al., 2008). Finalmente, la cafeína, presente en diversas bebidas, incluido café, té y colas, y chocolate, es otra de las substancias de amplio consumo en muchos países, debido a sus efectos psicoestimulantes, aunque con escasa frecuencia se estudia en las mujeres embarazadas (Kuczkowski, 2009).

La mayoría de los estudios sobre el consumo de substancias durante el embarazo se han centrado en la investigación de sus efectos en el feto y recién nacido, ya comentado, y en los factores de riesgo asociados, como un bajo nivel de educación, multiparidad, soltería y sintomatología ansiosa y depresiva (Alvarez-Segura et al., 2014; Cook et al., 2017; Havens, Simmons, Shannon y Hansen, 2009; Skagerstróm, Chang y Nilsen, 2011). Sin embargo, pocos han sido los estudios que se han focalizado en la personalidad como factor de riesgo del consumo de substancias en el embarazo.

Los rasgos de personalidad son importantes a la hora de explicar diferencias individuales en la conducta, cognición y emoción, son parcialmente heredables (Vukasovic y Bratko, 2015) y se sabe que están relacionados con la salud (Strickhouser et al., 2017). Investigaciones en población general indican que un elevado puntaje en los rasgos de personalidad de extraversión y neuroticismo se asocian al consumo adictivo (Connor-Smith y Flachsbart, 2010; Munafo, Zetteler y Clark, 2007). Los resultados del estudio del registro de gemelos y hermanos australianos muestran que un perfil de alto neuroticismo, baja cordialidad y responsabilidad se asocia a trastornos por uso sustancias, tabaco, alcohol, y cannabis, sin diferencias de sexo (Dash, Slutske, Martin, Statham, Agrawal y Lynskey, 2019). Por otro lado, se ha observado que la personalidad es un factor importante en la conducta materna y que un bajo neuroticismo y elevada cordialidad, extraversión y responsabilidad se asocian a una conducta materna adaptativa (McCabe, 2014). 
Parece que las mujeres que fuman durante el embarazo son aquellas que tienen con mayor frecuencia un perfil caracterizado por un alto neuroticismo, y búsqueda de novedad y baja extraversión (Lupattelli, Ronningen, Krogsrud, Noredeng y Ystrom, 2018; Massey et al., 2016; Maxson, Edwards, Ingram y Miranda, 2012), aunque un estudio lo asocia a un bajo neuroticismo (Ystrom, Vollrath y Nordeng, 2012). Mientras que aquellas con baja evitación del daño y alta autodirección son mas capaces de dejar de fumar (Massey et al., 2016), las que continúan consumiendo alcohol durante el embarazo puntúan alto en extraversión (Lupatelli et al., 2018) y apertura a experiencias (Beijers, Burger et al., 2014) y bajo en responsabilidad (Beijers et al., 2014; Ystrom et al., 2012).

Identificar los rasgos de personalidad y otros factores de riesgo asociados al consumo de substancias durante el embarazo parecería importante para prevenir el consumo y mejorar la salud materna y fetal/neonatal/infantil durante el embarazo y posparto.

El objetivo de este estudio fue evaluar las dimensiones de personalidad de Eysenk (1985) asociadas al consumo de substancias durante el embarazo, en una muestra de mujeres de población general que no hubieran requerido atención psiquiátrica o psicológica durante el embarazo, teniendo en cuenta la presencia de otros factores de riesgo, socio-demográficos, obstétricos, antecedentes psiquiátricos personales y familiares y grado de apoyo social.

\section{Material y Métodos}

\section{Participantes}

Durante el periodo de un año se incluyeron mujeres que acudieron al Servicio de Obstetricia y Ginecología de siete hospitales generales universitarios de España y que dieron a luz un niño vivo. El estudio forma parte de un proyecto (DEPREPOST) más amplio en el que se realizaba un estudio prospectivo desde el posparto inmediato hasta 32 semanas posparto (Sanjuan et al., 2008). Todas las participantes eran españolas, mayores de 18 años, caucásicas; ninguna había estado bajo tratamiento psiquiátrico/ psicológico durante el embarazo, y todas podían entender y contestar los cuestionarios clínicos. Se solicitó el consentimiento informado a todas las mujeres participantes. El estudio fue aprobado por los Comités de Ética de cada institución.

\section{Evaluación}

En el segundo o tercer día postparto, todas las participantes contestaban una entrevista semi-estructurada que incluía cuestiones socio-demográficas (edad, nivel de educación, empleo durante el embarazo, situación económica), reproductivas y obstétricas (planificación embarazo, paridad, tipo de parto, patología durante el embarazo), relación con la pareja y madre (ausencia pareja/madre, relación próxima y cálida, desavenencias esporádicas, o escasa relación), antecedentes psiquiátricos familiares y personales (se descartó mujeres que hubieran presentado cualquier condición mental que hubiera requerido tratamiento farmacológico o psicológico durante el embarazo) y consumo de substancias durante el embarazo.

El término consumo de substancias incluía tanto el uso de substancias legales (cafeína, tabaco y alcohol), como ilegales (cannabis, cocaína, estimulantes, alucinógenos, ketamina, éxtasis y opioides). Las mujeres fueron clasificadas como consumidoras o no consumidoras de substancias (aquellas que refirieron no consumir ninguna substancias lícita o ilícita durante el embarazo del hijo índice recién nacido). Las mujeres consumidoras de substancias además describieron la frecuencia usual de cada sustancia consumida durante el embarazo como: «nunca», «menos de una vez al mes», «mensual», «semanal», y «diario». Para el análisis, las primeras dos categorías («nunca», «menos de una vez al mes») se consideraron «nunca» y las mujeres fueron adscritas al grupo no consumidor. Por último las mujeres fueron invitadas a describir la dosis consumida durante el último mes de embarazo. Se utilizó como medida de cafeína una taza de café/día (equivalente a dos de té o tres bebidas de cola), de alcohol el número de bebidas estándar/ día, de tabaco el número de cigarrillos/día, y de cannabis el número de porros/día.

Por último, se administró a todas las mujeres el cuestionario de personalidad de Eysenck en su versión revisada corta EPQ-RS (Eysenck, 1985) para evaluar la personalidad. El EPQ-RS consiste en 48 ítems seleccionados entre los 100 ítems de la versión original que evalúan tres dimensiones de personalidad: extraversión $(\mathrm{E})$, neuroticismo $(\mathrm{N})$ y psicoticismo $(\mathrm{P})$. Extraversión o dimensión social que incluye los factores: sociabilidad, vivacidad, actividad, asertividad, búsqueda de sensaciones, despreocupación, espontaneidad, y aventurero; neuroticismo o dimensión emocional: ansiedad, depresión, sentimientos de culpa, baja autoestima, temor, irracionalidad, timidez, tristeza, y emotividad; y psicoticismo o dimensión impulsiva: agresividad, frialdad, impulsividad, egocentrismo, ausencia de empatía, antisocial, creatividad, y rigidez. En el presente estudio, se utilizaron las puntuaciones $\mathrm{T}$ de acuerdo a la versión estandarizada para la población española de mujeres (Eysenck y Eysenck, 2001).

\section{Análisis estadístico}

El análisis estadístico fue realizado utilizando los programas SPSS (v19.0) y R (v3.4.3). El test de chi-cuadrado fue utilizado para comparar las madres consumidoras y no consumidoras con respecto a la distribución de las variables categóricas. La comparación respecto a las variables continuas se realizó mediante la prueba $t$-de Student. Se definió un valor $p$ de 0,05 como estadísticamente significativo. El tamaño del efecto se ha cuantificado mediante la 
$d$ de Cohen en el caso de las variables numéricas y la $w$ de Cohen en el caso de las variables categóricas (Mangiafico, 2016). Para determinar qué factores están asociados con el consumo de substancias durante el embarazo, se usó un modelo multivariante de regresión logística. Para la selección de variables se siguió la propuesta de Hosmer y Lemeshow (Hosmer y Lemeshow, 2000), que incluye, en un primer paso, aquellas variables con un $p$-valor $<0,2$ en el análisis univariado así como aquellas variables que se consideraron relevantes por la literatura. Se exploró además la presencia de interacciones entre las variables. Se utilizó el test de Hosmer y Lemeshow para evaluar la bondad del ajuste del modelo de regresión logística. Los resultados se muestran como Odds ratio (ORs) con el intervalo de con- fianza del 95\% (IC95\%). En el caso de las dimensiones del EPQ incluidas en el modelo, se estimaron los OR asociados a una diferencia de 10 puntos en la puntuación.

\section{Resultados}

La tabla 1 muestra los datos de las variables socio-demográficas, reproductivas y obstétricas, de relación con la pareja y madre, la presencia de antecedentes psiquiátricos personales y familiares de las 1804 mujeres que cumplieron los criterios de inclusión del estudio. Las puntuaciones $T$ medias $(D E)$ de las dimensiones de personalidad fueron $51,1(9,59)$ para extraversión, 43,6 $(8,49)$ para neuroticismo y $48(8,93)$ para psicoticismo.

Tabla 1. Características de la muestra

\begin{tabular}{|c|c|c|c|c|c|c|c|c|c|c|c|}
\hline \multirow[b]{3}{*}{ Edad } & \multirow[b]{3}{*}{ Rango:18-46 } & \multirow{2}{*}{\multicolumn{2}{|c|}{$\begin{array}{c}\begin{array}{c}\text { Muestra total } \\
N=1804\end{array} \\
N(\%) / \bar{x}(D E)\end{array}$}} & \multirow{2}{*}{\multicolumn{2}{|c|}{$\begin{array}{l}\begin{array}{l}\text { Consumidoras } \\
N=909 \\
(50,4 \%)\end{array} \\
N(\%) / \bar{x}(D E)\end{array}$}} & \multirow{2}{*}{\multicolumn{2}{|c|}{$\begin{array}{c}\begin{array}{c}\text { No consumidoras } \\
\mathrm{N}=895(49,6 \%)\end{array} \\
N(\%) / \bar{x}(D E)\end{array}$}} & \multirow{3}{*}{$\begin{array}{c}\mathbf{x}^{2} / \mathbf{t} \\
0,321\end{array}$} & \multirow{3}{*}{$\begin{array}{l}\text { g.d.l. } \\
1802\end{array}$} & \multirow{3}{*}{$\begin{array}{c}\text { p valor } \\
0,749\end{array}$} & \multirow{3}{*}{$\begin{array}{c}\text { d-Cohen/ } \\
\text { w-Cohen }\end{array}$} \\
\hline & & & & & & & & & & & \\
\hline & & 31,80 & 4,65 & 31,80 & 4,53 & 31,70 & 4,78 & & & & \\
\hline \multirow[t]{3}{*}{ Nivel de educación } & Primaria & 560 & 31,1 & 310 & 31,4 & 250 & 28,1 & 12,588 & 2 & 0,002 & 0,08 \\
\hline & Secundaria & 743 & 41,3 & 378 & 41,6 & 365 & 41,0 & & & & \\
\hline & Universidad & 495 & 27,5 & 220 & 24,2 & 275 & 30,9 & & & & \\
\hline Estado civil & Casado/Pareja estable & 1732 & 96,5 & 880 & 96,9 & 852 & 96,1 & 2,317 & 3 & 0,509 & 0,04 \\
\hline \multirow[t]{4}{*}{ Situación laboral embarazo } & Empleado & 1217 & 67,8 & 596 & 65,6 & 621 & 70,0 & 8,414 & 3 & 0,038 & 0,07 \\
\hline & Sin empleo & 222 & 12,4 & 108 & 11,9 & 114 & 12,9 & & & & \\
\hline & Estudiante/Ama de casa & 165 & 9,2 & 93 & 10,2 & 72 & 8,1 & & & & \\
\hline & Baja por enfermedad & 192 & 10,7 & 112 & 12,3 & 80 & 9,0 & & & & \\
\hline \multirow[t]{4}{*}{ Situación económica } & Ingresos periódicos & 1115 & 62,8 & 569 & 63,1 & 546 & 62,5 & 4,246 & 3 & 0,236 & 0,04 \\
\hline & Dificultades ocasionales & 505 & 28,5 & 256 & 28,4 & 249 & 28,5 & & & & \\
\hline & Algunos problemas & 137 & 7,7 & 64 & 7,1 & 73 & 8,4 & & & & \\
\hline & Serios problemas & 18 & 1,0 & 13 & 1,4 & 5 & 0,6 & & & & \\
\hline \multirow[t]{3}{*}{ Embarazo planificado } & Planeado y contenta & 1208 & 74,6 & 613 & 72,8 & 595 & 76,5 & 2,884 & 2 & 0,237 & 0,04 \\
\hline & No planeado y contenta & 353 & 21,8 & 196 & 23,3 & 157 & 20,2 & & & & \\
\hline & Accidente & 59 & 3,6 & 33 & 3,9 & 26 & 3,3 & & & & \\
\hline Paridad & Primípara & 827 & 47,0 & 383 & 43,1 & 444 & 51,1 & 11,001 & 1 & 0,001 & 0,08 \\
\hline Patología durante el embarazo & $\mathrm{Si}$ & 292 & 16,2 & 184 & 20,2 & 108 & 12,1 & 21,617 & 1 & $<0,001$ & 0,11 \\
\hline \multirow[t]{5}{*}{ Relación con la pareja } & Sin pareja & 15 & 0,9 & 8 & 1,0 & 7 & 0,9 & 10,307 & 4 & 0,036 & 0,08 \\
\hline & Cercana y cálida & 1008 & 62,3 & 494 & 58,7 & 514 & 66,2 & & & & \\
\hline & Desacuerdos esporádicos & 546 & 33,8 & 314 & 37,3 & 232 & 29,9 & & & & \\
\hline & Mala relación & 16 & 1,0 & 8 & 1,0 & 8 & 1,0 & & & & \\
\hline & Tensa & 32 & 2,0 & 17 & 2,0 & 15 & 1,9 & & & & \\
\hline \multirow[t]{5}{*}{ Relación materna } & Sin madre & 84 & 5,2 & 52 & 6,2 & 32 & 4,1 & 7,256 & 4 & 0,123 & 0,07 \\
\hline & Cercana y cálida & 982 & 60,7 & 504 & 59,9 & 478 & 61,6 & & & & \\
\hline & Desacuerdos esporádicos & 491 & 30,3 & 260 & 30,9 & 231 & 29,8 & & & & \\
\hline & Mala relación & 51 & 3,2 & 20 & 2,4 & 31 & 4,0 & & & & \\
\hline & Tensa & 10 & 0,6 & 6 & 0,7 & 4 & 0,5 & & & & \\
\hline Historia familiar psiquiátrica & Si & 575 & 34,7 & 326 & 36,1 & 249 & 32,9 & 1,778 & 1 & 0,182 & 0,03 \\
\hline Historia psiquiátrica personal & $\mathrm{Si}$ & 299 & 16,6 & 170 & 18,7 & 129 & 14,4 & 5,692 & 1 & 0,017 & 0,06 \\
\hline EPQ-RS Extraversion (E) & & 51,1 & 9,59 & 51,5 & 9,22 & 50,7 & 9,93 & 1,793 & 1.802 & 0,073 & 0,08 \\
\hline EPQ-RS Neuroticismo (N) & & 43,6 & 8,49 & 44,0 & 8,44 & 43,2 & 8,53 & 1,804 & 1.802 & 0,071 & 0,09 \\
\hline EPQ-RS Psicoticismo (P) & & 48 & 8,93 & 48,7 & 9,15 & 47,2 & 8,64 & 3,644 & 1.802 & $<0,001$ & 0,17 \\
\hline
\end{tabular}

Nota. DE: Desviación estándar; EPQ-RS: Eysenck Personality Questionnaire Short Scale. 
María Luisa Imaz, Ricard Navinés, Estel Gelabert, Francina Fonseca, Alfonso Gutierrez-Zotes, Roser Guillamat, Klaus Langohr, Francesca Canellas, Inmaculada Gornemann, Javier Costas, Fermín Mayoral, Vicens Valles, Yolanda de Diego, Miquel Roca,

José Luis Iborra, Mónica Gratacos, Elisabeth Vilella, Lluïsa García-Esteve, Julio Sanjuan, Marta Torrens, Rocio Martin-Santos

Novecientas nueve mujeres $(50,4 \%)$ refirieron consumir alguna sustancia durante el embarazo, siendo la mayoría substancias legales: $40 \%$ cafeína, $20,8 \%$ tabaco, y el $3,5 \%$ alcohol. El consumo de substancias ilegales fue mucho menor $(1,5 \%)$, siendo dentro de estas la más frecuente el cannabis $(0,3 \%)$. La frecuencia de consumo de cafeína fue diaria en 577 (32\%) mujeres, semanal en 128 (7,1\%), y mensual en $21(1,2 \%)$ durante el embarazo. La frecuencia de consumo de tabaco fue diario en 348 (19,3\%) mujeres, semanal en $15(0,8 \%)$, y mensual en $13(0,7 \%)$. La frecuencia de consumo de alcohol durante el embarazo fue diario en una mujer $(0,1 \%)$, semanal en $23(1,3 \%)$, y mensual en $39(2,2 \%)$. La frecuencia de consumo de cannabis fue diaria en $4(0,2 \%)$ mujeres, semanal en $1(0,1 \%)$, y mensual en $1(0,1 \%)$. La dosis media $(D E)$ de cafeína en el último mes fue de 18,1 (24,5) tazas de café con un rango de (1-150); la de tabaco fue de 197 (171) cigarrillos (1-1120); la de alcohol fue de 29,7 (113) bebidas estándar (1-800); y la de cannabis de 42,2 (33,9) porros (1-90).

El resultado del análisis univariado se presenta en la tabla 1. Las mujeres que refirieron consumir substancias durante el embarazo respecto a las no consumidoras tenían un nivel de estudios menor $(p=0,002)$, un mayor porcentaje de desempleo durante el embarazo $(p=0,038)$, eran con mayor frecuencia multíparas $(p=0,001)$, habían tenido problemas médicos durante el embarazo $(p<0,001)$, tenían una pobre relación con su pareja $(p=0,05)$, y una mayor frecuencia de antecedentes psiquiátricos personales $(p<0,017)$. En relación a los rasgos de personalidad, las consumidoras de substancias mostraron puntuaciones más elevadas de extraversión y neuroticismo y de psicoticismo $(p=0,001)$ que las no consumidoras durante el embarazo, aunque estas dos primeras dimensiones sin alcanzar la significación estadística. Hay que señalar que el tamaño del efecto fue en todos los casos pequeño. No se observaron otras diferencias estadísticamente significativas entre ambos grupos de mujeres.

La tabla 2 muestra el modelo de regresión logística final de los factores predictivos de consumo de substancias durante el embarazo. Un menor nivel de educación $(p=0,046)$, estar de baja durante el embarazo $(p=0,008)$, y la presencia de antecedentes psiquiátricos previos al embarazo $(p=0,025)$ fueron factores independientes asociados al consumo. El ser primípara fue un factor protector $(p=$ $0,001)$. Las dimensiones de personalidad, extraversión ( $p=$ $0,029)$ y psicoticismo ( $p=0,009)$, fueron identificadas como factores de riesgo para el consumo de substancias durante el embarazo. Para cada incremento de 10 unidades en la puntuación de la dimensión de extraversión y psicoticismo del EPQ-RS, el odds de consumo de substancias durante el embarazo se incrementó un $12 \%$ y un $16 \%$ respectivamente tras ajustar por edad, nivel de educación, situación laboral, paridad, patología durante embarazo, relación con pareja/madre, y antecedentes psiquiátricos. El modelo final mostró un ajuste satisfactorio $(p=0,26)$.

\section{Discusión}

Este estudio explora las dimensiones de personalidad de Eysenck (1985) y el consumo de substancias, legales e ilegales, durante el embarazo en una muestra de $1804 \mathrm{mu}-$ jeres españolas de población general que acuden a dar a luz a su hospital general de la red pública de salud. Más de la mitad de la muestra estudiada había consumido alguna substancia durante el embarazo siendo la cafeína la substancia más frecuente, seguida del tabaco y del alcohol. El consumo de substancias ilícitas durante el embarazo fue muy poco frecuente, siendo el cannabis la substancia más habitual. Los resultados del estudio confirman que existe una moderada asociación entre la extraversión y el psicoticis-

Tabla 2. Modelo de regresión logística para predecir el consumo de substancias durante el embarazo

\begin{tabular}{|c|c|c|c|c|c|}
\hline Variables & & B (ES) & Wald & $\mathbf{p}$ & OR (IC 95\%) \\
\hline Constante & & $-1,656(0,424)$ & $-3,903$ & $<0,001$ & \\
\hline \multicolumn{6}{|c|}{ Nivel de educación (referencia: Universidad) } \\
\hline & Secundaria & $0,165(0,124)$ & 1,329 & 0,184 & $1,18(0,92-1,50)$ \\
\hline & Primaria & $0,275(0,138)$ & 1,991 & 0,046 & $1,32(1,00-1,73)$ \\
\hline \multicolumn{6}{|c|}{ Situación laboral maternal durante el embarazo } \\
\hline & Empleada & $0,249(0,158)$ & 1,571 & 0,116 & $1,28(0,94-1,75)$ \\
\hline & Estudiante/Ama de casa & $0,366(0,220)$ & 1,665 & 0,096 & $1,44(0,94-2,22)$ \\
\hline & Baja laboral & $0,555(0,211)$ & 2,633 & 0,008 & $1,74(1,15-2,63)$ \\
\hline Historia psiquiátrica personal & & $0,307(0,137)$ & 2,247 & 0,025 & $1,36(1,04-1,78)$ \\
\hline Primípara & & $-0,337(0,101)$ & $-3,324$ & 0,001 & $0,71(0,59-0,87)$ \\
\hline \multicolumn{6}{|l|}{ EPQ-RS } \\
\hline & Psicoticismo & $0,015(0,006)$ & 2,616 & 0,009 & $1,16(1,04-1,30)$ * \\
\hline
\end{tabular}

Nota. ES: Error estándard; OR: Odds ratio; IC: Intervalo de confianza del 95\%; EPQ-RS: Eysenck Personality Questionnaire Short Scale.

* Odds ratios asociados a una diferencia de 10 puntos en las puntuaciones de las dimensiones del EPQ-RS. Bondad de ajuste de Hosmer-Lemeshow: $\mathrm{p}=0,26$. 
mo y el consumo de substancias durante el embarazo. Otros factores independientes relacionados con el consumo fueron un bajo nivel educativo, estar de baja laboral durante el embarazo, la multiparidad y la presencia de antecedentes psiquiátricos personales previos al embarazo.

\section{Prevalencia de consumo de sustancias legales e ilegales durante el embarazo}

En cuanto al consumo de substancias lícitas durante el embarazo, los datos de prevalencia del consumo de cafeína en la literatura son muy limitados a pesar de ser la substancia psicoactiva más ampliamente consumida en el mundo (Kuczkowski, 2009). En nuestra muestra, un $40 \%$ de las mujeres embarazadas tomaban de media 1-2 tazas de café al día, acercándose a los niveles descritos de cafeína (> 200mg/dia) que se asocian con efectos negativos sobre el embarazo y el periodo perinatal (Chen, Bell, Browne, Druschel y Romitti, 2014; Modzelewska et al., 2019), recomendándose reducir la ingesta de cafeína al mínimo durante el embarazo (Jahanfar y Jaafar, 2013).

En relación al consumo de tabaco durante el embarazo, los datos de población general en nuestro país (Puig et al., 2012), son semejantes a los de nuestro estudio y confirmados por una revisión sistemática reciente (Lange et al., 2018). La prevención del consumo de tabaco debería iniciarse antes del embarazo, y mantenerse a lo largo del mismo, enfocada a la mujer y su entorno familiar (WHO, 2013).

No podemos descartar que la relativa baja prevalencia de consumo de alcohol observada en el presente estudio sea debido en parte a que las mujeres lo hayan ocultado. Las estimaciones de consumo de tabaco durante el embarazo son más exactas que las de alcohol u otras substancias (Chamberlain et al., 2013). El alcohol es una sustancia conocida con efectos teratogénicos, y probablemente epigenéticos, y su consumo prenatal y gestacional es causa de morbilidad materna y fetal (Cook et al., 2017). Un aspecto importante del consumo de alcohol durante el embarazo es el de los embarazos no planificados (WHO, 2014), presente en la cuarta parte de las mujeres de nuestra muestra. Resulta difícil delimitar la relación dosis efecto por lo que la recomendación es abstenerse de consumir alcohol preferentemente desde la etapa preconcepcional cuando se planifica el embarazo (Hoyne et al., 2016; WHO, 2014).

Con respecto al consumo de cannabis, estudios en $\mathrm{mu}-$ jeres embarazadas (Ebrahim y Gfroerer, 2003; Kassada, Marcon, Pagliarani y Rossi, 2013) estiman un porcentaje de consumo de cannabis entre un 1,5-2,8\%, semejante a nuestros resultados. Es posible que factores psicológicos y sociales influencien las mujeres a discontinuar el consumo de algunas substancias más que otras, y que la percepción de dañar al feto sea diferente con las substancias ilegales que con las legales (Mark y Terplan, 2017). En el momento actual es importante estar atentos al papel de la legaliza- ción de consumo de cannabis en muchos países y a su uso y prevención durante el embarazo (Scheyer et al., 2019).

\section{Dimensiones de personalidad y otros factores asociados al consumo de substancias en el embarazo}

Los resultados del estudio indican que las variables de personalidad deberían ser tenidas en cuenta en la valoración de riesgos de consumo de substancias en las mujeres embarazadas. La extraversión, dimensión que refleja sociabilidad, energía, asertividad, búsqueda de sensaciones y despreocupación, predice también riesgo de consumo en nuestra muestra tras ajustar por otros factores de riesgo. Durante el embarazo se ha observado que las mujeres extravertidas tienen mayor tendencia a continuar bebiendo alcohol (Ystrom et al., 2012). La probable explicación de estos resultados sería que estas mujeres toman substancias para disfrutar en situaciones sociales, en línea con investigaciones que muestran que las personas extravertidas beben para incrementar los sentimientos positivos más que para evitar los sentimientos negativos (Kuntsche et al., 2008; Munafo et al., 2007). Esta necesidad de estimulación puede por otro lado ayudar a desestimar el riesgo que implica para la salud el consumo y favorezca que la mujer continúe consumiendo durante el embarazo. Estudios sobre el consumo de tabaco durante el embarazo (Maxson et al., 2012) señalan que las fumadoras muestran niveles bajos de extraversión en comparación con las no fumadoras. Un reciente estudio multinacional en 18 países europeos, utilizando un cuestionario anónimo «on line», ha explorado el impacto de las características de personalidad de la mujer sobre los hábitos de beber y fumar antes y durante el embarazo (Lupatelli et al., 2018). Mujeres con alto nivel de extraversión presentaban una tendencia $10-17 \%$ mayor a consumir niveles medios/elevados de alcohol, mientras que un elevado neuroticismo se asociaba a un incremento del riesgo del $16 \%$ de continuar fumando durante el embarazo (Connor-Smith y Flachsbart, 2007). Los resultados mostraron asociaciones rasgo-específicas en determinadas regiones europeas.

En la muestra estudiada no se observa una asociación entre neuroticismo y consumo de substancias durante el embarazo. En teoría, el neuroticismo ha sido relacionado con un estilo de afrontamiento focalizado en la emoción y alejado del riesgo de daño (ej. utilización consumo de substancias para enfrentarse a emociones) y empíricamente se correlaciona (con un tamaño del efecto medio) con consumo de substancias como vía de enfrentarse al estrés (Connor-Smith y Flachsbart, 2007). Investigaciones previas han asociado el consumo de tabaco como vía de afrontar las emociones y problemas, con elevado riesgo de continuar fumando durante el embarazo (Lopez, Konrath y Seng, 2011). Aunque hay datos que apuntan que no hay diferencias de género en conseguir abstenerse de fumar (Marqueta, Nerín, Gargallo y Beamonte, 2017), el embarazo puede 
ser una importante motivación en las mujeres para conseguirlo. Un reciente estudio realizado en mujeres embarazadas ha encontrado que las fumadoras con bajo nivel de la dimensión evitación del daño y elevada autodirección mostraban ser más capaces de abstenerse del consumo durante el embarazo (Massey et al., 2016).

Por último, la asociación entre psicoticismo y consumo de substancias durante el embarazo puede ser atribuido a que esta dimensión está relacionada con la impulsividad y con medir escaso razonamiento y prioridad (Eysenck, 1985; Kuntsche et al., 2008). La impulsividad ha sido considerada un factor de vulnerabilidad para el consumo de substancias (Vassileva y Conrod, 2019), e incluso algunas teorías de la adicción la conceptualizan como un síndrome del control de los impulsos (Conrod y Nicolau, 2016). De hecho, la triada búsqueda de sensaciones, ansiedad, e impulsividad se han encontrado asociadas de forma repetida con el consumo de substancias (Crews y Boettiger, 2009; Ersche, Turton, Pradhan, Bullmore y Robbins, 2010). Los datos apoyan la hipótesis de que las mujeres embarazadas con alto psicoticismo/impulsividad presentan mayor riesgo de continuar consumiendo substancias psicoactivas durante el embarazo.

Confirmando los datos previos de la literatura, el consumo de substancias durante el embarazo se asoció de forma independiente además con un bajo nivel educativo (Havens et al., 2009), situación de baja laboral (Truong, Lupattelli, Kristensen y Nordeng, 2017), y la presencia de historia personal psiquiátrica (Conway, Compton, Stinson y Grant, 2006; EMCDDA, 2012). Por el contrario, ser madre primípara fue un factor protector. Estar embarazada por primera vez puede ser una motivación intensa para el cambio o para modificar conductas. Las mujeres primíparas tienen más éxito a la hora de dejar el alcohol y el tabaco durante el embarazo (Powers et al., 2013; Schneider et al., 2010).

\section{Limitaciones}

Este estudio tiene varias potenciales limitaciones que tienen que ser consideradas a la hora de interpretar los resultados. El consumo de substancias ha sido recogido mediante una entrevista semi-estructurada y puede ser susceptible de presentar sesgos. Cabe señalar el escaso consumo ilegal autoreferido. Se han descrito diversos factores asociados a una menor prevalencia de consumo, como el miedo a las consecuencias legales, sentimientos de culpa, fallos de memoria y método de la entrevista (Friguls et al., 2012; Shipton et al., 2009). En este sentido, la evaluación del consumo se realizó a los 2-3 días del parto cuando las madres conocían el estado de salud de sus hijos recién nacidos. Por otro lado, la frecuencia del consumo fue preguntada sobre cada mes del embarazo mientras que la cantidad de consumo se refirió al último mes de embarazo. Se puede argumentar que esta cifra puede no ser representativa de la cantidad de substancias consumidas en el total del embarazo. Muchas mujeres consumidoras habituales que dejan el consumo lo hacen el primer trimestre, por lo que parece importante evaluarlo en este momento (Shipton et al., 2009; Figueras et al., 2008). El consumo fue recogido de forma retrospectiva y el diseño transversal no nos permite establecer la dirección de la asociación. Nuestro estudio no recogió datos sobre las mujeres no consumidoras expuestas al humo del tabaco durante el embarazo (Aurrekoetxea et al., 2014).

Respecto a la personalidad hubiera sido mejor explorarla antes del embarazo en un marco más neutro que el del 2-3 ${ }^{\circ}$ día posparto. Sería interesante explorar otras dimensiones de personalidad y de estilos de afrontamiento.

Los resultados del estudio no pueden ser generalizados al total de la población general ya que excluimos las mujeres embarazadas con trastorno psiquiátrico actual, que en sí mismo es un factor de riesgo asociado al consumo de substancias durante el embarazo (Figueras et al., 2008). Por último, queremos señalar que se trata de una muestra extensa de población no clínica en nuestro país que estudia los factores de personalidad y su asociación con consumo de substancias durante el embarazo y que tiene en cuenta otros factores de riesgo.

\section{Conclusiones}

En una muestra no clínica, una de cada dos mujeres refirió haber consumido substancias psicoactivas, en su mayoría cafeína, alcohol y tabaco, durante el embarazo. El consumo de substancias se asoció a características socio-demográficas, antecedentes de historia psiquiátrica, problemas de salud en el embarazo y rasgos de personalidad. Los resultados indican que las dimensiones del extraversión y psicoticismo (mujeres con elevada sociabilidad, energía, búsqueda de sensaciones y asertividad, mayor impulsividad y menor reflexión) tienen un impacto en el consumo de tabaco, alcohol y cafeína durante el embarazo.

La identificación temprana de los factores de riesgo debería incluir el estudio de los rasgos de personalidad. El cribaje sistematizado de consumo de tóxicos legales e ilegales debería ser preconcepcional, y repetido cada trimestre durante el embarazo en aquellas mujeres con resultados positivos (urinoanálisis/autoinformes) o con factores de riesgo. Ambas medidas ayudarán a establecer programa de prevención e intervención para prevenir los efectos del consumo de substancias sobre la diada madre-hijo.

\section{Reconocimientos}

Este trabajo ha sido realizado con las ayudas de investigación del Instituto de Investigación Carlos III números de referencia FIS: PI041635, PI041783, PI041758, PI041761, PI041791, PI041766 y PI041782, así como con la Red de Genotipación y Psiquiatría Genética: GO3/184. También 
queremos agradecer el apoyo para grupos de investigación consolidados. SGR2014/1114; SGR2017/1798; 2017 SGR2017/622; al Centro de Investigación Biomédica en Red en Salud Mental (CIBERSAM); a la Red de Asistencia Primaria (RediAPP); y a la Red de Trastornos Adictivos (RETIC). Nuestro agradecimiento a todas las mujeres que participaron en el estudio.

\section{Aspectos éticos}

El estudio ha sido aprobado por los Comités Éticos y de Investigación (CEICs) de los centros participantes (Hospital Clínic e IMIM-Hospital del Mar de Barcelona; Hospital Pere Mata, URV Reus; Hospital Consorci Sanitari de Terrasa; Corporació Sanitaria Parc Tauli de Sabadell; Hospital Son Dureta, Palma de Mallorca; Hospital Carlos Haya y Fundación IMABIS, Málaga; y Hospital Clínico, UV, Valencia). Todos los procedimientos realizados se han llevado a cabo de acuerdo con la declaración de Helsinki de 1964 y posteriores modificaciones, así como con los estándares éticos y de investigación, nacionales y de las instituciones participantes. Se obtuvo el consentimiento informado por escrito de todas las participantes.

\section{Conflictos de interés}

Ninguno de los autores declara conflictos de interés.

\section{Contribución de los autores}

MLI, RN, LG, MT y RMS, diseño del estudio. MLI, RN y $\mathrm{KL}$, análisis de los datos. MLI, RN, EG y RMS, escribieron la primera versión del manuscrito. AG, RG, VV, FC, MR, IG, FM, EG, RMS y JS llevaron a cabo la recogida de los datos. MT, FF y LG tuvieron un papel relevante en la interpretación de los resultados. Todos los autores participaron y aprobaron la última versión del manuscrito.

\section{Referencias}

Alshaarawy, O. y Anthony, J. C. (2019). Cannabis use among women of reproductive age in the United States: 20022017. Addictive Behaviors, 99, 106082. doi:10.1016/j.addbeh.2019.106082.

Alvarez-Segura, M., Garcia-Esteve, L., Torres, A., Plaza, A., Imaz, M. L., Hermida-Barros, L., ... Burtchen, N. (2014). Are women with a history of abuse more vulnerable to perinatal depressive symptoms? A systematic review. Archives of Women's Mental Health, 17, 343-357. doi:10.1007/s00737-014-0440-9.

Ait-Daoud, N., Blevins, D., Khanna, S., Sharma, S., Holstege, C. P. y Amin, P. (2019). Women and Addiction: An Update. Medical Clinics of North America, 103, 699-711. doi:10.1016/j.mcna.2019.03.002
Aurrekoetxea, J. J., Murcia, M., Rebagliato, M., Fernández-Somoano, A., Castilla, A. M., Guxens, M., ... Santa-Marina, L. (2014). Factors associated with second-hand smoke exposure in non-smoking pregnant women in Spain: Self-reported exposure and urinary cotinine levels. The Science of the Total Environment, 470-471, 1189-1196. doi:10.1016/j.scitotenv.2013.10.110.

Beijers, C., Burger, H., Verbeek, T., Bockting, C. L. H. y Ormel, J. (2014). Continued smoking and continued alcohol consumption during early pregnancy distinctively associated with personality. Addictive Behaviors, 39, 980986. doi:10.1016/j.addbeh.2014.01.022.

Bhuvaneswar, C. G., Chang, G., Epstein, L. A. y Stern, T. A. (2008). Cocaine and opioid use during pregnancy: prevalence and management. Primary Care Companion to The Journal of Clinical Psychiatry, 10, 59-65. doi:65. 10.4088/ pcc.v10n0110.

Chamberlain, C., O’Mara-Eves, A., Porter, J., Coleman, T., Perlen, S. M., Thomas, J. y Mckenzie, J. E. (2017). Psychosocial interventions for supporting women to stop smoking in pregnancy. Cochrane Database of Systematic Reviews, 2, CD001055. doi:10.1002/14651858.CD001055. pub.

Chen, P. y Jacobson, K. C. (2012). Developmental trajectories of substance use from early adolescence to young adulthood: Gender and racial/ethnic differences. Journal of Adolescent Health, 50, 154-163. doi:10.1016/j.jadohealth.2011.05.013.

Chen, L., Bell, E. M., Browne, M. L., Druschel, C. M., Romitti, P. A. y National Birth Defects Prevention Study. (2014). Exploring Maternal Patterns of Dietary Caffeine Consumption Before Conception and During Pregnancy. Maternal and Child Health Journal, 18, 2446-2455. doi:10.1007/s10995-014-1483-2.

Connor-Smith, J. K. y Flachsbart, C. (2007). Relations Between Personality and Coping: A Meta-Analysis. Journal of Personality and Social Psychology, 93, 1080-1107. doi:10.1037/0022-3514.93.6.1080.

Conrod, P. J. y Nikolaou, J. (2016). Annual Research Review: On the developmental neuropsychology of substance use disorders. Child Psychology and Psychiatry, 57, 371-394. doi: 10.1111/jcpp.12516.

Conway, K. P., Compton, W., Stinson, F. S. y Grant, B. F. (2006). Lifetime comorbidity of DSM-IV mood and anxiety disorders and specific drug use disorders: Results from the National Epidemiologic Survey on Alcohol and Related Conditions. Journal of Clinical Psychiatry, 67, 247-257.

Cook, J. L., Green, C. R., de la Ronde, S., Dell, C. A., Graves, L., Ordean, A., .... Wong, S. (2017). Epidemiology and effects of substance use in pregnancy. Journal of Obstetrics and Gynaecology Canada, 39, 906-915. doi: 10.1016/j. jogc.2017.07.005. 
María Luisa Imaz, Ricard Navinés, Estel Gelabert, Francina Fonseca, Alfonso Gutierrez-Zotes, Roser Guillamat, Klaus Langohr, Francesca Canellas, Inmaculada Gornemann, Javier Costas, Fermín Mayoral, Vicens Valles, Yolanda de Diego, Miquel Roca,

José Luis Iborra, Mónica Gratacos, Elisabeth Vilella, Lluïsa García-Esteve, Julio Sanjuan, Marta Torrens, Rocio Martin-Santos

Crews, F. T. y Boettiger, C. A. (2009). Impulsivity, frontal lobes and risk for addiction. Pharmacology Biochemistry and Behavior, 93, 237-247. doi:10.1016/j.pbb.2009.04.018.

Dash, G. F., Slutske, W. S., Martin, N. G., Statham, D. J., Agrawal, A. y Lynskey, M. T. (2019). Big Five personality traits and alcohol, nicotine, cannabis, and gambling disorder comorbidity. Psychology of Addictive Behaviors, 33, 420-429. doi:10.1037/adb0000468.

Díaz-Mesa, E. M., García-Portilla, P., Fernández-Artamendi, S., Sáiz, P. A., Bobes Bascarán, T., Casares, M. J. y Bobes, J. (2016). Diferencias de género en la gravedad de la adicción. Adicciones, 28, 221-230. doi:10.20882/adicciones.829.

Ebrahim, S. H. y Gfroerer, J. (2003). Pregnancy-related substance use in the United States during 1996-1998. Obstetrics and Gynecology, 101, 374-379. doi:10.1016/ S0029-7844(02)02588-7.

Ersche, K. D., Turton, A. J., Pradhan, S., Bullmore, E. T. y Robbins, T. W. (2010). Drug addiction endophenotypes: Impulsive versus sensation-seeking personality traits. Biological Psychiatry, 68, 770-773. doi:10.1016/j. biopsych.2010.06.015.

European Monitoring Centre for Drugs and Drug Addiction [EMCDDA]. (2012). Pregnancy, childcare and the family: key issues for Europe's response to drugs. Recuperado de http://www.emcdda.europa.eu/publications/selected-issues/children_bg.

European Monitoring Centre for Drugs and Drug Addiction [EMCDDA]. (2016). European Drug Report 2016: trends and developments. Recuperado de http: //www. emedda.europa. eu/publications / edr/trends-developments/2016_en.

Eysenck, H. (1985). Personality and individuals differences. A natural science approach. New York: Plenun Press.

Eysenck, H. y Eysenck, S. (2001). Cuestionario revisado de personalidad de Eysenck: versiones completa (EPQ-R) y abreviada (EPQ-RS). Madrid: TEA.

Eysenck, S. (1985). Eysenck Personality Questionnaire-Revised $(E P Q-R)$ and Short-Scale (EPQ-RS). London: Hooder and Stoughton.

Figueras, F., Meler, E., Eixarch, E., Francis, A., Coll, O., Gratacos, E. y Gardosi, J. (2008). Association of smoking during pregnancy and fetal growth restriction: Subgroups of higher susceptibility. European Journal of Obstetrics Gynecology and Reproductive Biology, 138, 171-175. doi:10.1016/j.ejogrb.2007.09.005..

Friguls, B., Joya, X., Garcia-Serra, J., Gómez-Culebras, M., Pichini, S., Martinez, S., ... Garcia-Algar, O. (2012). Assessment of exposure to drugs of abuse during pregnancy by hair analysis in a Mediterranean island. Addiction, 107, 1471-1479. doi:10.1111/j.1360-0443.2012.03828.x.

Havens, J. R., Simmons, L. A., Shannon, L. M. y Hansen, W. F. (2009). Factors associated with substance use during pregnancy: Results from a national sample. Drug and Alcohol Dependence, 99, 89-95. doi:10.1016/j.drugalcdep.2008.07.010.

Hosmer, D., y Lemeshow, S. (1989). Applied Logistic Regression. New York: Wiley \& Sons.

Hoyme, H. E., Kalberg, W. O., Elliott, A. J., Blankenship, J., Buckley, D., Marais, A-S., ... May, P. A. (2016). Updated clinical guidelines for diagnosing fetal alcohol spectrum disorders. Pediatrics, 138. doi:10.1542/peds.2015-4256.

Jahanfar, S. y Jaafar, S. H. (2013). Effects of restricted caffeine intake by mother on fetal, neonatal and pregnancy outcome. Cochrane Database of Systematic Reviews, 2, CD006965. doi:10.1002/14651858.CD006965.pub3.

Kassada, D. S., Marcon, S. S., Pagliarini, M. A. y Rossi, R. M. (2013). Prevalence of drug abuse among pregnant women | Prevalência do uso de drogas de abuso por gestantes. Acta Paulista de Enfermagem, 26, 467-471.

Kuntsche, E., von Fischer, M. y Gmel, G. (2008). Personality factors and alcohol use: A mediator analysis of drinking motives. Personality and Individual Differences, 45, 796-800. doi:10.1016/j.paid.2008.08.009.

Kuczkowski, K. M. (2009). Caffeine in pregnancy. Archives of Gynecology and Obstetrics, 280, 695-698. doi: 10.1007/ s00404-009-0991-6.

Lamy, S., y Thibaut, F. (2010). Psychoactive substance use during pregnancy: A review | État des lieux de la consommation de substances psychoactives par les femmes enceintes. Encephale, 36, 33-38. doi:10.1016/j.encep.2008.12.009.

Lange, S., Probst, C., Rehm, J. y Popova, S. (2017). Prevalence of binge drinking during pregnancy by country and World Health Organization region: Systematic review and meta-analysis. Reproductive Toxicology, 73, 214221. doi:10.1016/j.reprotox.2017.08.004.

Lange, S., Probst, C., Rehm, J. y Popova, S. (2018). National, regional, and global prevalence of smoking during pregnancy in the general population: A systematic review and meta-analysis. The Lancet Global Health, 6, e769776. doi: 10.1016/S2214-109X(18)30223-7.

Louw, K. A. Substance use in pregnancy (2018). The medical challenge. Obstetric Medicine, 11, 54-66. doi:10.1177/173495X17750299.

Lupattelli, A., Ronningen, E., Krogsrud, S. K., Noredeng, H. y Ystrom, E. (2018). Personality and its relation to the use of alcohol and cigarettes during pregnancy: a multinational study. Journal of Health Psychology, 1, 1-14. doi:10.1177/1359105318775194.

Mangiafico, S. S. (2016). Summary and analysis of extension program evaluation in $R$, version 1.18.1. Recuperado en https://www.rcompanion.org/handbook/. (Pdf version: rcompanion.org/documents/RHandbookProgramEvaluation.pdf).

McCabe, J. R. (2014). Maternal personality and psychopathology as determinants of parenting behavior: A quan- 
titative integration of two parenting literatures. Psycholologycal Bulletin, 140, 722-750. doi:10.1037/a0034835.

Mark, K. y Terplan, M. (2017). Cannabis and pregnancy: Maternal child health implications during a period of drug policy liberalization. Preventive Medicine, 104, 46-49. doi:10.1016/j.ypmed.2017.05.012.

Marqueta, A., Nerín, I., Gargallo, P. y Beamonte, A. (2017). Gender differences in success at quitting smoking: short- and long-term outcomes. Adicciones, 29, 13-21. doi:10.20882/adicciones.826.

Massey, S. H., Reiss, D., Neiderhiser, J. M., Leve, L. D., Shaw, D. S. y Ganiban, J. M. (2016). Maternal personality traits associated with patterns of prenatal smoking and exposure: Implications for etiologic and prevention research. Neurotoxicology and Teratology, 53, 48-54. doi:10.1016/j.ntt.2015.11.010.

Maxson, P. J., Edwards, S. E., Ingram, A. y Miranda, M. L. (2012). Psychosocial differences between smokers and non-smokers during pregnancy. Addictive Behaviors, 37, 153-159. doi:10.1016/j.addbeh.2011.08.011.

Modzelewska, D., Bellocco, R., Elfvin, A., Brantsæter, A. L., Meltzer, H. M., Jacobsson, B. y Sengpiel, V. (2019). Caffeine exposure during pregnancy, small for gestacional age birth and neonatal outcome_results from the Norwegian Mother and Child Cohort Study. BMC Pregnancy and Childbirth, e19, 80. doi:10.1186/s12884019-2215-9.

Munafò, M. R., Zetteler, J. I. y Clark, T. G. (2007). Personality and smoking status: A meta-analysis. Nicotine and Tobacco Research, 9, 405-413. doi:10.1080/14622200701188851.

Patró-Hernández, R.M., Nieto Robles, Y. y Limiñana-Gras, R.M. (2019). The relationship between Gender Norms and Alcohol Consumption: A Systematic Review. Adicciones, 32, 145-158doi:10.20882/adicciones.1195.

Popova, S., Lange, S., Probst, C., Gmel, G. y Rehm, J. (2017). Estimation of national, regional, and global prevalence of alcohol use during pregnancy and fetal alcohol syndrome: a systematic review and meta-analysis. The Lancet Global Health, 5, e290-e299. doi:10.1016/ S2214-109X(17)30021-9.

Powers, J. R., McDermott, L. J., Loxton, D. J. y Chojenta, C. L. (2013). A prospective study of prevalence and predictors of concurrent alcohol and tobacco use during pregnancy. Maternal and Child Health Journal, 17, 76-84. doi:10.1007/s10995-012-0949-3.

Puig, C., Vall, O., García-Algar, Ó., Papaseit, E., Pichini, S., Saltó, E. y Villalbí, J. R. (2012). Assessment of prenatal exposure to tobacco smoke by cotinine in cord blood for the evaluation of smoking control policies in Spain. BMC Pregnancy and Childbirth, 12, 26. doi:10.1186/14712393-12-26.

Sanjuán, J., Martin-Santos, R., Garcia-Esteve, L., Carot, J. M., Guillamat, R., Gutierrez-Zotes, A., ... De Frutos, R. (2008). Mood changes after delivery: Role of the sero- tonin transporter gene. British Journal of Psychiatry, 193, 383-388. doi:10.1192/bjp.bp.107.045427.

Schneider, S., Huy, C., Schütz, J. y Diehl, K. (2010). Smoking cessation during pregnancy: A systematic literature review. Drug and Alcohol Review, 29, 81-90. doi:10.1111/ j.1465-3362.2009.00098.x.

Scheyer, A. F., Melis, M., Trezza, V. y Manzoni, O. J. J. (2019). Consequences of perinatal cannabis exposure. Trends in Neurosciences, 42, 871-884. doi:10.1016/j. tins.2019.08.010.

Shipton, D., Tappin, D. M., Vadiveloo, T., Crossley, J. A., Aitken, D. A. y Chalmers, J. (2009). Reliability of self reported smoking status by pregnant women for estimating smoking prevalence: A retrospective, cross sectional study. British Medical Journal, 339, b4347. doi:10.1136/ bmj.b4347.

Skagerstróm, J., Chang, G. y Nilsen, P. (2011). Predictors of drinking during pregnancy: A systematic review. Journal of Women's Health, 20, 901-913. doi:10.1089/ jwh.2010.2216.

Strickhouser, J. E., Zell, E. y Krizan, Z. (2017). Does personality predict health and well-being? A meatasynthesis. Health Psychology, 36, 797-810. doi: 10.1037/hea0000475.

Substance Abuse and Mental Health Services Administration [SAMHSA]. (2013). Results from the 2012 National Survey on Drug Use and Health (NSDUH) Volume 1: Summary of National Findings. Rockville: Substance Abuse and Mental Health Services Administration. Recuperado de http://www.samhsa.gov/data/sites/default/file/ NSDUHresults2012/NSDUH results2012.pdf.

Truong, B. T., Lupattelli, A., Kristensen, P. y Nordeng, H. (2017). Sick leave and medication use in pregnancy: A European web-based study. BMJ Open, 7, e014934. doi:10.1136/bmjopen-2016-014934.

Vassileva, J. y Conrod, P. J. (2019). Impulsivities and addictions: a multidimensional integrative framwork informing assessment an interventions for substance use disorders. Philosophical Transactions of the Royal Society B: Biologycal Sciences, 374, 20180137. doi:10.1098/ rstb.2018.0137.

Volkow, N. D., Compton, W. M. y Wago, E. M. (2017). The risks of marijuana use during pregnancy. JAMA, 317,129-130.

Vukasovic, T. y Bratko, D. (2015). Heritability of personality: a meta-analysis of behaviour genetic studies. Psychologycal Bulletin, 141, 769-785. doi: 10.1037/bul0000017.

World Health Organization [WHO]. (2013). WHO recommendations for the prevention and management of tobacco use and second-hand smoke exposure in pregnancy. Geneva: World Health Organization. Recuperado en https:// apps.who.int/iris/bitstream/handle/10665/94555/ 9789241506076_eng.pdf;sequence=1.

World Health Organization [WHO]. (2014). Guidelines for identification and management of substance use and substan- 
María Luisa Imaz, Ricard Navinés, Estel Gelabert, Francina Fonseca, Alfonso Gutierrez-Zotes, Roser Guillamat, Klaus Langohr, Francesca Canellas, Inmaculada Gornemann, Javier Costas, Fermín Mayoral, Vicens Valles, Yolanda de Diego, Miquel Roca,

José Luis Iborra, Mónica Gratacos, Elisabeth Vilella, Lluïsa García-Esteve, Julio Sanjuan, Marta Torrens, Rocio Martin-Santos

ce use disorders in pregnancy. Geneva, Switzerland: World Health Organization. Recuperado en http://www.who. int/substance_abuse/publications/pregnancy-guidelines/cn/.
Ystrom, E., Vollrath, M. E. y Nordeng, N. (2012). Alcohol, and cigarettes during pregnancy. European Journal of Clinical Pharmacology, 68, 845-851. doi:10.1007/s00228-011$1197-\mathrm{y}$. 